\title{
Customized Assisted Reproduction Enhancement (CARE) for Women with Extremely Poor Ovarian Reserve (EPOR)
}

\author{
Jan Tesarik* \\ MAR Gen Clinic Granada, Spain
}

Submission: March 09, 2017; Published: April 05, 2017

*Corresponding author: Jan Tesarik, MD, PhD, MAR Gen Clinic, Camino de Ronda 2, 18006 Granada, Spain, Email: jtesarik@clinicamargen.com

\begin{abstract}
The ovarian reserve is usually evaluated by determining serum levels of anti-Müllerian hormone (AMH) and ovarian antral follicle count. It is widely accepted that women with moderately impaired ovarian reserve (AMH levels between $0.2 \mathrm{ng} / \mathrm{ml}$ and $1.1 \mathrm{ng} / \mathrm{ml}$ ) have a poor chance of having a child by in vitro fertilization (IVF) because of a low response to ovarian stimulation. However, conflicting data have been published as to predicting the chances of women with extremely poor ovarian reserve ( $\mathrm{AMH} \leq 0.2 \mathrm{ng} / \mathrm{ml}$ ). In many clinics in the world women with extremely poor ovarian reserve (EPOR) are not admitted for IVF assuming that their chance of success is zero.
\end{abstract}

This report shows that a relatively high clinical pregnancy rate $(23 \%)$ and delivery rate $(18 \%)$ can be achieved in this category of women by using customized assisted reproduction enhacement (CARE). In the CARE protocol the patient treatment is personally tailored during the pre-stimulation phase, ovarian stimulation, the embryological laboratory work and the patient follow-up after embryo transfer.

Keywords: Customized treatment; Poor ovarian reserve; Low responder; Assisted reproduction; Anti-Müllerian hormone; IVF in europe

\section{Introduction}

Ovarian reserve is a term that refers to the quantity and quality of the pool of ovarian non-growing follicles (NGF) which can be recruited for growth and yield viable oocytes [1]. The ovarian reserve, represented by several million NGF at birth, diminishes progressively with the age, mainly due to follicle death by apoptosis until menopause is reached at an average age of 50-51 years, with approximately 1,000 NGF left. Even though the reduction of the ovarian reserve is age-related, there is significant interindividual variability in its timing. Consequently, the actual size of the NGF pool cannot be accurately predicted by the woman's age.

Several predictors of the ovarian reserve have been suggested. Recently, serum levels of anti-Müllerian hormone (AMH) are used as the most common marker. The consensus meeting of the European Society of Human Reproduction and Embryology (ESHRE) working group on poor ovarian reserve (POR), held in 2011 in Bologna, suggested the serum AMH level of $1.1 \mathrm{ng} / \mathrm{ml}$ as cutoff to be used for POR diagnosis [2]. Within the group of POR women, those with AMH levels $\leq 0.2 \mathrm{ng} / \mathrm{ml}$ are considered to have extremely poor ovarian reserve (EPOR) [3].

There are controversial opinions about the chance of women with EPOR in an eventual IVF attempt. In spite of the fact that one study has reported that women with EPOR still have $4.4 \%$ ongoing pregnancy rates per treatment cycle and $16 \%$ cumulative ongoing pregnancy rates after repeated cycles, other authors could not establish any pregnancies in 26 women up to 44 years of age with $\mathrm{AMH}$ concentrations $\leq 0.15 \mathrm{ng} / \mathrm{ml}$ using a range of different treatment approaches [4]. In many European countries women with EPOR are not admitted for IVF by infertility clinics, in particular in public hospitals.

The present report describes a high ongoing pregnancy and delivery rate in women with EPOR achieved with the use of a series of rules and measures, named "Customized Assisted Reproduction Enhancement" (CARE). According to the CARE protocol, the treatment of EPOR patients is systematically adapted to the individual condition of each of them at different phases of the treatment, including the period preceding the beginning of ovarian stimulation, the ovarian stimulation, embryological laboratory work, and the patient follow-up after embryo transfer.

\section{Patients and Methods}

This study involves 78 treatment cycles, performed in 78 women with $\mathrm{AMH}$ levels $\leq 0.2 \mathrm{ng} / \mathrm{ml}$ in the period between January 2012 and March 2016. The women's mean age was 37 
years, ranging between 32 and 40 years. If a treatment attempt was repeated in the same woman, only the first attempt is included in this study, and cumulative success rates are not presented. Among the women actually treated with CARE protocol in this period, those over 40 years of age and those with endometriosis are not included in this report.

Previously described methods for ovarian stimulation, oocyte and embryo handling and embryo transfer [5] were used with small modifications, as described below. The GnRH antagonist protocol was used in all cases. IVF was performed by intracytoplasmic sperm injection (ICSI).

\section{CARE Protocol}

The customization of the treatment protocol for women with EPOR concerns different subsequent phases of the treatment, starting 1-3 months before the beginning of ovarian stimulation, the ovarian stimulation itself, laboratory work related to IVF, embryo culture and handling, and the period after embryo transfer including the first three months of pregnancy (Table 1). The special measures adopted in the CARE protocol are of two types: (1) those related to the condition of EPOR, and thus applied to all patients in this condition, and (2) those tailored to each individual patient, and thus variable from patient to patient. Table 1: Basic characteristics of the CARE protocol.

\begin{tabular}{|c|c|c|}
\hline Phase of Treatment & Common Measures & $\begin{array}{l}\text { Patient-Tailored } \\
\text { Measures }\end{array}$ \\
\hline \multirow{3}{*}{ Pre-stimulation } & DHEA treatment & $\begin{array}{l}\text { Optimization of } \\
\text { uterine cavity }\end{array}$ \\
\hline & Contraceptive pill & HCG priming \\
\hline & Estradiol priming & \\
\hline \multirow{2}{*}{ Ovarian stimulation } & GH co-stimulation & $\begin{array}{c}\text { Individualized FSH/ } \\
\text { LH ratio }\end{array}$ \\
\hline & Low-dose aspirin & $\begin{array}{c}\text { Timing of HCG } \\
\text { trigger }\end{array}$ \\
\hline $\begin{array}{c}\text { Gamete \& embryo } \\
\text { handling }\end{array}$ & None & $\begin{array}{l}\text { Laser-assisted } \\
\text { hatching }\end{array}$ \\
\hline \multirow[t]{2}{*}{ Post embryo transfer } & Frequent checkups & $\begin{array}{c}\text { Flexible luteal phase } \\
\text { support }\end{array}$ \\
\hline & GnRH agonist & \\
\hline
\end{tabular}

The common measures in the pre-stimulation phase included the treatment with dehydroepiandrosterone (DHEA), at the daily dose of $75 \mathrm{mg}$ split in three $25 \mathrm{mg}$ doses, during 6-8 weeks preceding the onset of ovarian stimulation, one cycle of contraceptive pill before the stimulation, and 3-5 days oral estradiol priming ( $1 \mathrm{mg}$ estradiol daily) from day one of the cycle following the pill until the beginning of the stimulation. The uterine cavity was evaluated in each woman by conventional or virtual hysteroscopy, and eventual pathologies were corrected when necessary [6]. The injection of 10,000 IU HCG was done in cases with very low plasma testosterone levels $(<0.3 \mathrm{ng} / \mathrm{ml})$ during the cycle preceding ovarian stimulation (Table 1 ).

In the ovarian stimulation phase, growth hormone (GH) and low-dose aspirin were used, together with gonadotropins, in all patients as described [7]. Other aspects of the stimulation regimen were flexible, adapted to the patient's response to treatment. The ratio of FSH and LH activities in the exogenous gonadotropin formulas used for ovarian stimulation was continuously adapted during stimulation according to repeated measures of serum estradiol and LH levels, and the part of the LH component was increased when the LH values fell below 1 IU/l [8]. The timing of the HCG trigger with respect to the time of ovarian puncture was decided individually, and ranged between $36 \mathrm{~h}$ and $37 \mathrm{~h}$, according to the size of the antral follicles on the day of trigger. When one or more medium-sized follicles were present, in addition to a large follicle, a second HCG trigger was applied approximately $24 \mathrm{~h}$ before ovarian puncture in order to facilitate the release of cumulus-oocyte complexes from these smaller follicles.

As to the laboratory work for ICSI, the deformation of the oocyte zona pellucida under the pressure of the injection needle was evaluated while the first oocyte was being injected. In case of excessive deformation, without needle penetration, the injection procedure was halted and all available oocytes were subsequently injected by laser-assisted ICSI [9].

Particular attention was paid to the period after embryo transfer with frequent hormonal controls throughout the whole first trimester. Serum concentrations of estradiol and progesterone were determined on the day of embryo transfer and then every 3 days until the pregnancy test. Hormonal supplementation of the luteal phase [5] was continuosly adapted according to the last hormone measures, with daily doses of orally administered estradiol and vaginally administered progesterone going up to $6 \mathrm{mg}$ and $800 \mathrm{mg}$, respectively, in cases in which the serum concentrations of these hormones tended to decrease. In addition, GnRH agonist was used for luteal phase support in all cases $[5,10]$.

\section{Results and Discussion}

The results of IVF obtained with the CARE protocol are summarized in Table 2. Out of the 78 started treatment cycles, 2 were canceled before ovarian puncture because of the lack of response to stimulation, and no oocytes were recovered by ovarian puncture in other 2 women. In one case the cycle had to be canceled because of fertilization failure. In the remaining 73 cases embryo transfer was performed (Table 2). The treatment resulted in 18 clinical pregnancies and 14 deliveries, corresponding to clinical pregnancy rate of $23 \%$ and delivery rate of $18 \%$ per started treatment cycle. All deliveries resulted in the birth of a single child, corresponding to live birth rate of $13 \%$ (Table 2). Out of the 78 women included in this study, 41 had previously been denied IVF treatment in other clinics in Spain, France and Italy, claiming that egg donation was the only way they can get pregnant. The present results demonstrate the feasibility of childbirth with one's own eggs in this condition, although the probability of birth is low (23\%) as compared to $80 \%$ in our current egg donation program. 
Table 2: Treatment outcomes with the CARE protocol.

\begin{tabular}{|c|c|}
\hline Numbers & \\
\hline A. Attempts started & 78 \\
\hline B. Attempts canceled without ovarian puncture & 2 \\
\hline C. Ovarian punctures with no oocyte recovered & 2 \\
\hline D. Attempts with oocyte recovered but no embryo transfer & 1 \\
\hline E. Attempts with at least one embryo transferred & 73 \\
\hline F. Embryos transferred & 105 \\
\hline G. Attempts with positive b-HCG & 21 \\
\hline $\begin{array}{c}\text { H. Attempts with biochemical pregnancy (no gestational } \\
\text { sac) }\end{array}$ & 3 \\
\hline I. Attempts with clinical pregnancy (gestational sac \\
detected) & 18 \\
\hline J. Abortions & 4 \\
\hline K. Attempts with delivery & 14 \\
\hline L. Number of babies born & 14 \\
\hline Rates & $18 \%$ \\
\hline Clinical pregnancy rate (I/A) & $13 \%$ \\
\hline Delivery rate (K/A) & \\
\hline Birth rate (L/F) & $18 \%$ \\
\hline
\end{tabular}

The success rates achieved with the CARE protocol are relatively high for this patient condition, as compared with other studies dealing with women in a similar situation $[3,11]$. CARE puts together different treatments and measures, some of which, when applied alone, have been shown previously to improve IVF results in women with POR.

In the pre-stimulation period, DHEA improves ovarian function, increases pregnancy chances and, by reducing aneuploidy, lowers miscarriage rates in POR patiens [12]. Steroid hormone priming is believed to help synchronize the population of recruitable follicles before the start of stimulation [13]. We also observed an improvement of endometrial growth with this treatment, especially in women whose serum estradiol levels increased slowly in response to ovarian stimulation. HCG priming has been shown to improve the response to ovarian stimulation in patients with previous IVF failures [14]. With the use of conventional or virtual hysteroscopy [6] we have also detected different anomalies of the uterine cavity in many patients of this group. Consequently, these anomalies were corrected before the start of ovarian stimulation.

During the ovarian stimuation, co-treatment with $\mathrm{GH}$ improves success rates in women aged $>40$ years [7] and in younger women with multiple IVF failures [15], whereas lowdose aspirin alone does not appear to have any beneficial effect [16]. Tailoring the $\mathrm{FSH} / \mathrm{LH}$ ratio in the formula of the mix of gonadotropins used for ovarian stimulation according to the current serum LH levels is known to improve oocyte yield and quality [8]. During ICSI we have often observed unusually high zona pellucida resistance in this group of patients. We have reported previously that laser-assisted ICSI reduces oocyte damage in this condition [9]. Moreover, the hole created in the zona pellucida during this procedure may facilitate the subsequent hatching of the respective embryos.

The follow-up of women after embryo transfer is very important in the condition of EPOR, since we often observed abnormally low levels of progesterone, and to a lesser extent estradiol, just a few days after transfer. These abnormalities were corrected immediately by enhancing the luteal phase support with higher doses of exogenous hormones. Most of the patients who needed this early correction of the luteal phase support would probably never get pregnant without this early intervention. Some patients showed unexpected falls in progesterone level even later during the first trimester of pregnancy. We believe that frequent hormonal controls, carried out short after embryo transfer and during early pregnancy, can avoid implantation failure and abortion in many cases. The importance of adequate early luteal phase support, including the use of GnRH agonist [5], has been highlighted recently [10].

\section{Conclusion}

CARE is a protocol with which acceptable success rates can be obtained in women with EPOR, considered to be one of the conditions with the worst prognosis for IVF treatment at present. The possibility of using CARE in other, less severe cases of female infertility in order to improve IVF outcomes is a challenge for future clinical research.

\section{References}

1. Wallace WHB, Kelsey TW (2010) Human ovarian reserve from conception to the menopause. PLOS ONE 5(1): e8772.

2. Ferraretti AP, Marca LA, Fauser BC, Tarlatzis B, Nargund G, et al. (2011) ESHRE consensus on the definition of 'poor response' to ovarian stimulation for in vitro fertilization: the Bologna criteria. Hum Reprod 26(7): 1616-1624.

3. Kedem A, Haas J, Geva LL, Yerushalmi G, Gilboa Y, et al. (2013) Ongoing pregnancy rates in women with low and extremely low AMH levels. A multivariate analysis of 769 cycles. PLoS One 8(12): e81629.

4. Nelson SM, Yates RW, Lyall H, Jamieson M, Traynor I, et al. (2009) AntiMüllerian hormone-based approach to controlled ovarian stimulation for assisted conception. Hum Reprod 24(4): 867-875.

5. Tesarik J, Hazout A, Mendoza-Tesarik R, Mendoza N, Mendoza C (2006) Beneficial effect of luteal GnRH agonist administration on embryo implantation in both GnRH agonist-and antagonist-treated ovarian stimulation cycles. Hum Reprod 21(10): 2572-2579.

6. Tesarik J, Mendoza-Tesarik R, Mendoza N (2017) Virtual ultrasonographic hysteroscopy followed by conventional operative hysteroscopy, enabling pregnancy. Am J Obstet Gynecol 216(2): 188.e1.

7. Tesarik J, Hazout A, Mendoza C (2005) Improvement of delivery and live birth rates after ICSI in women aged $>40$ years by ovarian costimulation with growth hormone. Hum Reprod 20(9): 2536-2541.

8. Tesarik J, Mendoza C (2002) Effects of exogenous LH administration during ovarian stimulation of pituitary down-regulated young oocyte donors on oocyte yield and developmental competence. Hum Reprod 17(12): 3129-3137.

9. Rienzi L, Greco E, Ubaldi F, Iacobelli M, Martinez F, et al. (2001) Laserassisted intracytoplasmic sperm injection. Fertil Steril 76(5): 10451047. 
10. Tesarik J, Mendoza-Tesarik R, Mendoza N (2016) Gonadotropinreleasing hormone agonist for luteal phase support: the origin of the concept, current experience, mechanism of action and future perspectives. Fertil Steril 106(2): 268-269.

11. Jirge PR (2016) Poor ovarian reserve. J Hum Reprod Sci 9(2): 63-69.

12. Gleicher N, Barad DH (2011) Dehydroepiadrosterone (DHEA) supplementation in diminished ovarian reserve (DOR). Reprod Biol Endocrinol 9: 67.

13. Blockeel C, Engels S, De Vos M, Haentjens P, Polyzos NP, et al. (2012) Oestradiol valerate pretreatment in GnRH-antagonist cycles: a randomized controlled trial. Reprod Biomed Online 24(3): 272-280.
14. Beretsos P, Partsinevelos GA, Arabatzi E, Drakakis P, Mavrogianni D, et al. (2009) "hCG priming" effect in controlled ovarian stimulation through a long protocol. Reprod Biol Endocrinol 7: 91.

15. Hazout A, Junca AM, Ménézo Y, de Mouzon J, Cohen-Bacrie P (2009) Effect of growth hormone on oocyte competence in patients with multiple IVF failures. Reprod Biomed Online 18(5): 664-670.

16. Frattarelli JL, McWilliams GD, Hill MJ, Miller KA, Scott RT (2008) Lowdose aspirin use does not improve in vitro fertilization outcomes in poor responders. Fertil Steril 89(5): 1113-1117.

\section{Your next submission with Juniper Publishers} will reach you the below assets

- Quality Editorial service

- Swift Peer Review

- Reprints availability

- E-prints Service

- Manuscript Podcast for convenient understanding

- Global attainment for your research

- Manuscript accessibility in different formats

( Pdf, E-pub, Full Text, Audio)

- Unceasing customer service

Track the below URL for one-step submission https://juniperpublishers.com/online-submission.php 